\title{
Annotations in Digital Libraries and Collaboratories - Facets, Models and Usage
}

\author{
Maristella Agosti $^{1}$, Nicola Ferro ${ }^{1}$, Ingo Frommholz ${ }^{2}$, and Ulrich Thiel ${ }^{2}$ \\ 1 Department of Information Engineering, University of Padua, Italy \\ \{maristella.agosti,nicola.ferro\}@unipd.it \\ 2 Fraunhofer IPSI, Darmstadt, Germany \\ \{frommholz, thiel\}@ipsi.fraunhofer.de
}

\begin{abstract}
This paper presents the results of our study regarding the different facets and ways of using annotations in both digital libraries and collaboratories. This study represents an innovative attempt at gathering methodological tools and synergies from both fields in order to effectively define a comprehensive model for annotations. Thus we propose a conceptual model for annotations in order to develop an annotation service that can be plugged into digital libraries and collaboratories. Finally, starting from our model, we introduce a search strategy for exploiting annotations in order to search and retrieve relevant documents for a user query.
\end{abstract}

\section{Introduction}

The research field regarding the design and development of software systems, that are able to provide annotation capabilities on the content that they manage, e.g. digital libraries and collaboratories, is very active and productive. On the other hand the problem of how to incorporate annotations is usually faced separately in the field of digital libraries and collaboratories without exploiting the synergies that can be common to both fields. Our research work represents a first effort to face these issues together in both fields. This way we can benefit by the methodological tools coming from both fields in order to define a comprehensive model for annotations and to design an annotation service that can be seamlessly plugged into different digital libraries and collaboratories.

The paper is organised as follows: the remainder of this section presents digital libraries and collaboratories and the beneficial usage of annotations in those fields. Section 2 discusses different angles about annotations, Section 3 introduces our conceptual model for annotations and some access and retrieval strategies that exploit annotations; finally, Section 4 draws some conclusions and presents the future work.

\subsection{Digital Libraries and Collaboratories}

Digital libraries are not only the digital versions of traditional libraries, but offer means going beyond mere presentation of the content stored in a digital repository. Two definitions of digital libraries, coming from two different directions 
and thus focusing on different aspects, point to this fact. The more computer science oriented view is expressed in the introduction to the first issue of the International Journal on Digital Libraries (cited in [8]):

Digital Libraries are concerned with the creation and management of information resources, the movement of information across global networks and the effective use of this information by a wide range of users.

Librarians have a different definition of Digital Libraries:

Digital Libraries are organisations that provide the resources, including the specialised stuff, to select, structure, offer intellectual access to, interpret, distribute, preserve the integrity of, and ensure the persistence over time of collections of digital works so that they are readily and economically available for use by a defined community or set of communities. (Digital Library Federation (DLF), 1998, cited in [8])

Both these definitions highlight some distinguishing features of digital libraries: firstly the central point of both definitions is that information resources should be accessed and used; then they further couple this concept with the one of community of users. In this way a digital library is jointly characterised by its collection of information resources and by the community of users for whom the collection is managed and made available. Other aspects addressed by the above definitions are the creation and interpretation of resources. The two definitions share the common view that information resources have to be accessed with the last point addressed in the definition of a collaboratory formulated by William Wulf, who sees such a collaboratory as

...center without walls, in which nation's researchers can perform their research without regard to geographical location - interacting with colleagues, accessing instrumentation, sharing data and computation resource, and accessing information in digital libraries. [14]

Collaboratories focus on facilitating scientific interaction within a team. Besides this, they should support the sharing of data and resources. Figure 1 summarises the aspects of digital libraries and collaboratories. As we will discuss in the following, all these aspects are particularly relevant for annotations and they can greatly benefit from having annotations available as an additional tool.

\subsection{Annotations Within Digital Libraries}

Annotations can be exploited in order to realise the distinguishing features of digital libraries highlighted above. The creation of new information resources is supported by annotations in two ways. First, when users add annotations to existing information resources, these are new information resources themselves. Second, annotations can also assist in the creation of new information resources. Through annotations, new ideas and concepts can be discussed and the results of such a discussion can then be integrated into the newly created 


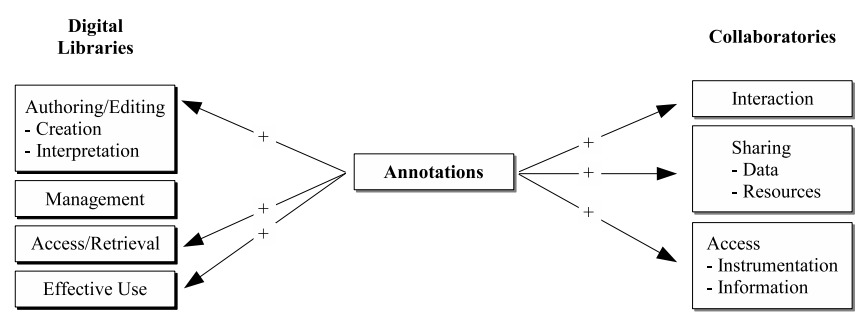

Fig. 1. Digital Libraries, Collaboratories and Annotations

object. Annotations might increase and expand the information resources managed by the digital library. In this way, they may provide interpretations of information resources. User communities benefit from such interpretations in that they help understanding the annotated resource and contain additional information about it. In the Humanities, for instance, interpretation is one of the basic tasks scholars perform. Systems like COLLATE or IPSA support this task through annotations $[1,7]$. Annotations support user communities in accessing the information resources provided by the digital library in a personalised and customisable way: indeed users can create annotations that link different documents, enabling alternative paths for browsing digital contents and thus structuring them in alternative ways, like virtual books [19]. Different layers of annotations can coexist in the same document: a private layer of annotations accessible only by the annotations author himself, a collective layer of annotations, shared by a team of people, and finally a public layer of annotations, accessible to all the users of the digital library; in this way user communities can benefit from different views of the information resources managed by the digital library $[16,15]$. Annotations can contain interpretations, reviews and additional information about the resources they belong to. They reflect what others say about a resource, which establishes an interesting context exploitable for information retrieval [7]. Furthermore the access and retrieval of information resources can be aided by means of automatic annotations. Employing topic detection techniques, a document can be segmented into topics of desired granularity and automatic annotations represent a summary of these topics. Then, exploiting automatic hypertext construction techniques [3], automatic annotations can be linked to the original document. Finally, the content of annotations can support the effective use of the digital resources. Automatic annotations, interpretations, alternative paths, and all other information contained in annotations help the user in approaching a document.

\subsection{Annotations Within Collaboratories}

As we pointed out above, the main characteristics of collaboratories are interaction, sharing and access. Annotations can be beneficial for all of them. Indeed, many systems use annotations to establish collaboration. Wilensky sees annotations as an example for spontaneous collaboration [23]. Interaction within a 
community can be supported by means of shared or public annotations. In COLLATE, annotations are used to model a scientific discourse between film scientists. The system supports strong collaboration through nested annotations; users can directly react to other users' contributions and do not have to rely on traditional means like e-mail or telephone [7]. Annotations are an important way to share one's results with others. Shared or public annotations are visible to more persons than the author who created them. Systems supporting data sharing through annotations are, among others, those reported in $[1,7,9,19]$. Sharing data triggers at least weak collaboration - users can view others' results, without necessarily directly reacting to them. The access aspect in collaboratories can be supported by annotations the same way as discussed in the last subsection.

\section{Annotations}

Since annotations intrinsically entail an active involvement of the users with information resources, they naturally bring digital libraries and collaboratories closer, so that it is advisable to investigate how to exploit methods and techniques coming from both fields in order to effectively employ annotations. Over the past years a lot of research work regarding annotations has been done [18,20], which led to different viewpoints about what an annotation is. The following sections describe the different angles about annotations that we consider.

\subsection{Annotations as Metadata}

Annotations are considered as additional data about an existing content, that is annotations are metadata [18]. This reflects a data specific view on annotations. From a syntactic point of view one of the main characteristics of metadata is that it is connected to the object it refers to; annotations have a similar connection to what they are annotating. This way, they are indeed data about data.

The World Wide Web Consortium (W3C) considers annotations as metadata and interprets them as the first step in creating an infrastructure that will handle and associate metadata with content towards the Semantic Web [11]; examples are the Annotea Project ${ }^{1}$ and the Extensible MultiModal Annotation (EMMA) ${ }^{2}$ markup language. Also systems that employ annotations as an extension of bookmarks can fall within this definition. Indeed the additional data provided by annotations are exploited to describe, organise, categorise and search the bookmarks [13]. As a further example, MPEG-7, named "Multimedia Content Description Interface" and developed by the International Organization for Standardization (ISO) ${ }^{3}$, is a standard for describing the multimedia content data to be processed by a device or a computer code. Finally also automatic annotations can be considered metadata, since they extract summary sentences or significant phrases from the document they annotate, thus providing additional data for highlighting the key-points of the document.

\footnotetext{
${ }^{1}$ http://www .w3.org/2001/Annotea/

2 http://www.w3.org/TR/2003/WD-emma-20031218/

${ }^{3}$ http://www.iso.ch/iso/en/prods-services/popstds/mpeg.html
} 


\subsection{Annotations as Content}

Another view on annotations is seeing them as content, reflecting an information specific view. Annotations can be regarded as content in two ways: they can be content about content and they can be considered as additional content [18]. Both ways do not mutually exclude each other: interpretations, for example, are content about content, but they might also contain additional content. Reviews and judgements, as another example, are basically content about content.

Annotations being additional content augment existing content and allow the creation of new relationships among existing contents, by means of links that connect annotations together and with existing content. In this sense we can consider that existing content and annotations constitute a hypertext, according to the definition of hypertext provided in [5]. For example, [17] considers annotations as a natural way of enhancing hypertexts by actively engaging users with existing content in a digital library [16].

Normally digital libraries do not have a hypertext connecting documents with each other; thus annotations can represent a means for associating an hypertext to a digital library. In this way it is then possible to exploit the associated hypertext in order to enjoy alternative browsing paths and to perform advanced document searches, employing hypertext information retrieval techniques [4].

\subsection{Annotations as Dialogue Acts}

Another viewpoint on annotations, regarding them as dialogue acts, covers a communication specific view. This view is concerned with the question of the pragmatics conveyed in annotations, i.e. the intention behind a user's statement. Gaining information about pragmatics is an important means to distinguish between the different kinds of content we have discussed in the last subsection. We may find out about the semantics of utterances in annotations, but this does not mean that we can distinguish whether we can see the annotation as content about content or an extension of existing content, or even something completely different. This distinction might be important when applying appropriate retrieval functions, as we will see in Section 3.3.

Each annotation implicitly consists of certain communicative acts, which, according to Searle can be classified as (among others) assertives, directives (e.g., requests), and commissives (e.g., promises) [22]. Communicative acts both allow for communication on the content and on the meta level. On the content level, assertives connected with a certain discourse structure relation are the units with which a coherent interpretation of the material can be created [6]. On the other hand, directives and commissives can trigger further collaborative acts on the meta level. Directives can be used to attempt to get some other person to do something; an example would be if a user asks the author of a comment if he could further elaborate on it. The author, in turn, can answer the request with a promise to provide the needed information (and actually provide it later on). Certain communicative acts can thus enable strong collaboration, and they can be realised as annotations. 


\section{Comprehensive Model of Annotations}

We aim to design and develop a comprehensive model for annotations able to address all the previously described facets and to define an appropriate strategy for exploiting annotations in searching and retrieving documents or other annotations.

\subsection{Design Choices}

Considering the complexity of the annotation and the need for a proper conceptual model of annotation, as explained above, we have decided to make the effort of modelling the annotation using a conceptual modelling tool of general use as the Entity-Relationship (ER) model is. As introduced in our previous work [2], in order to capture the complex semantics of the annotation, which emerged also from the discussion of Section 2, we can distinguish between the meaning and sign of annotations. The meaning of annotation is a main aspect concerning the concept of annotation, which identifies conceptual differences within the semantics of the annotation. For example the different angles about annotations introduced in Section 2 can be considered as different meanings of annotation. Furthermore, within a given angle, we can identify different meanings of annotation; for example, within the "annotation as content" viewpoint we can point out three different meanings of annotation: comprehension and study, interpretation and divulgation, and revision and cooperation. The sign of annotation is a way of representing a meaning of annotation. For example we can identify a textual or a graphic sign of annotation. These basic signs can be combined together in order to create a more complex sign of annotation, capable to express complex meanings of annotation, such as those explained above. Thus an annotation is expressed by one or more signs of annotation, that in turn are characterised by one or more meanings of annotation, defining the overall semantics of the annotation.

Before discussing the proposed conceptual schema, our reference architecture introduced in [2] has to be borne in mind: we aim to design and develop an annotation service that can be easily plugged into different digital libraries or collaboratories, allowing these systems to seamlessly extend their functionalities. As an important consequence of this architectural choice, we assume that the annotation service knows everything about annotations but it has no knowledge about documents managed by the system it is plugged into. This is due to the fact that the annotation service directly manages annotations while documents and information pertaining to them are provided by the system the annotation service is plugged into. Thus the annotation service deals with handles to document, that allow it to connect annotations to documents, without the need to actually manage them. 


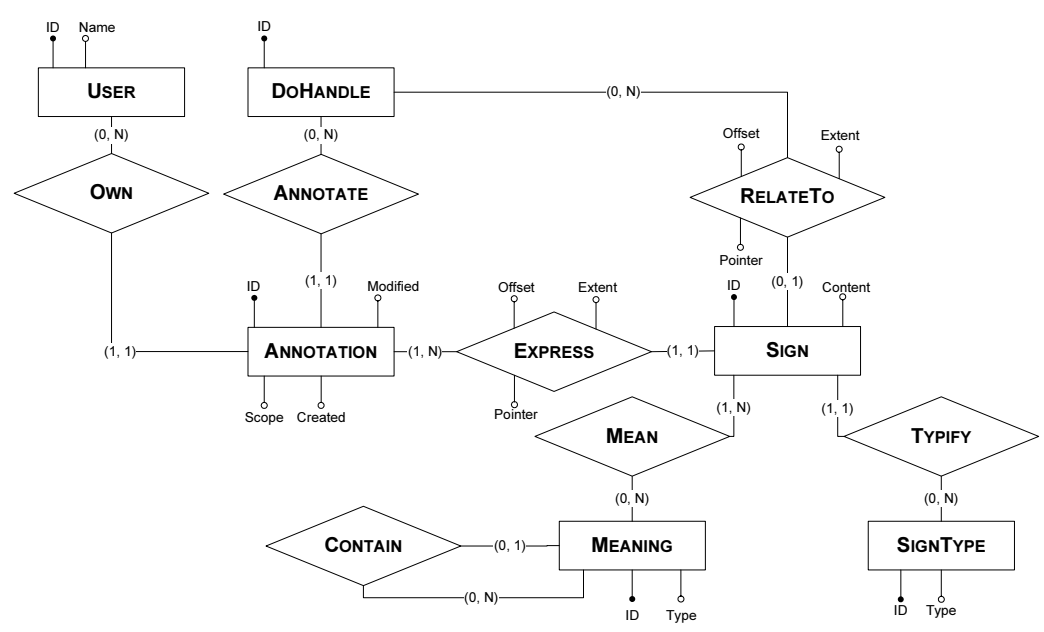

Fig. 2. Entity-Relationship schema for modelling annotations

\subsection{Annotation Conceptual Schema}

The proposed conceptual schema is shown in Figure 2. It is centred around two main issues: how to model annotations and how to connect them to information resources. The next sections describe these two issues in detail.

How to Model Annotations. The Annotation entity represents the abstraction of the annotation, i.e. it expresses the existence of an object capable of annotating another object, without further specifying its characteristic. This is the pivot entity, which provides the basis for modelling annotations. The ANNOTATION entity owns the following attributes: ID is a unique identifier for the annotation, e.g. an Uniform Resource Identifier (URI); Created and Modified represent, respectively, the creation date and the last modified date of the annotation; and Scope specifies if the annotation is private, shared by a team or public.

The discussion carried out in the previous sections showed that the ANNOTATION entity alone is not sufficient for covering the semantics of the general concept of annotation, so it needs to be partnered with two other entities MEANING and SIGN, representing respectively the meaning of annotation and the sign of annotation. The MEANING entity is characterised by a unique identifier, ID, and by a Type, which describes the meaning of annotations. On the MEANING entity there is a recursive relationship, CONTAIN, that expresses the existence of broader meanings and narrower meanings; thus the meanings of annotation can be organised in a simple hierarchy and some navigation facilities within this hierarchy can provided to the user. The CONTAIN relationship expresses the fact that a meaning may be contained only in one other meaning and that it may contain one or more other meanings. The SIGN entity owns an unique identifier, ID, and a Content attribute, which represent the actual content of the sign of 
annotation, e.g. a piece of text. The SignTyPe entity describes the kind of a sign of annotation, e.g. a textual sign or a graphic sign, and makes it possible to correctly interpret the Content attribute of a Sign. The SignTyPE entity is connected to the SIGN entity by means of the TYPIFY relationship, which expresses the fact that a Sign must have exactly one SignType, while a SignType may specify one or more Sign entities.

Two relationships, Express and MeAn, allow the three entities AnNotaTion, Meaning and Sign to cooperate together for defining the semantics and the materialisation of an annotation. The ExPREss relationship denotes that an Annotation entity have to be expressed at least by one Sign entity, and eventually more, and that a given Sign entity has to be employed in order to express one and only one AnNotation entity. The attributes of Express allow us to physically identify which part of the information resource has to be annotated. In particular the Pointer attribute identifies a portion of a digital object, e.g. it could be an XPath expression in case of an eXtensible Markup Language (XML) document; the Offset attribute selects a starting offset with respect to the portion identified by Pointer, e.g. the initial character within an XML element; finally the Extent attribute specifies the size of the sign of annotation, e.g. the number of characters that are annotated within the portion identified by Pointer starting from Offset.

The MEAN relationship expresses the fact that a Sign entity has to be related at least to one MEANing entity, and eventually more, and that a MEANing entity may characterise one or more Sign entities.

How to Connect Annotations to Information Resources. As explained in the previous section, the ANNOTATION entity represents the abstraction of an object capable of annotating another object. In order to connect annotations to information resources we need also an entity that represents the abstraction of an object that can be annotated; this entity is called DoHandLE, which represents a digital object by means of an handle to it. Thus the cornerstones for connecting annotations to information resources are the ANNOTATION and DoHANDLE entities which represents the fact that there are two kinds of related objects: digital objects that can be annotated and annotations that annotate those digital objects.

The relationship between annotations and annotated digital objects is represented by the ANNOTATE relationship, which links an ANNOTATION entity to the DoHANDLE entity it annotates. This relationship expresses the fact that an annotation must annotate one and only one digital object and that a digital object may be annotated by one or more annotations. Once we have annotated a digital object, the annotation itself can be considered as a digital object eligible to be annotated. Thus the conceptual schema has the additional constraint that, after that the annotation has been created, also an occurrence of the DoHANDLE entity corresponding to the annotation have to be added, in order to allow the newly created annotation to be annotated too. Users can therefore create not only sets of annotations concerning a digital object, but also threads of annotations - i.e. annotations in reply one to another - which are the basis for actively engaging users with the system and for enabling collaboration. 
The RELATETo relationship is used for the purpose of relating to other digital object and it associates a sign of an annotation with the digital object it refers to. This relationship holds between DoHANDLE and Sign and not between DoHAndle and Annotation, because the Annotation, perceived as abstraction, does not have to be related to a digital object, since its main purpose is to contribute to modelling the fact that there exist annotating objects and annotated object. On the contrary, the sign of annotation takes charge of relating to a digital object and the explanation of this relation is given by the meanings of annotation associated with that sign. The RELATETo relationship allows a SigN entity to refer or not to a digital object, while a digital object may be referred to by one or more signs of annotation. The attributes of RELATETo have the same meaning of the attributes of ExpRESS. On the whole the ExPRESS relationship specifies the origin of the link and the RELATETo relationship identifies the destination of the link.

Finally the USER entity represents a user, granted by the system. The OwN relationship relates an annotation with its author; a user may create one or more annotations, while an annotation must belong to one and only one user.

The proposed conceptual schema provides us a great flexibility, because we can express the different aspects of an annotation, couple them together and it does not constrain us to fixed types of annotations for fixed tasks. So our proposal represents an enhancement and a generalisation with respect to $[11,21]$; in fact being a conceptual schema, our model can be easily mapped to different models, such as a relational schema, a Resource Description Framework (RDF) schema or a XML schema; this way it provides us great flexibility with respect to different architectural choices.

\subsection{Search and Retrieval Issues}

Although annotations are quite a useful and common concept in digital libraries and collaboratories, there do not exist many retrieval approaches taking annotations into account. As one of the few examples, Golovchinsky et al. use annotations to construct full-text queries out of them [10]. What is missing so far are retrieval functions which are potentially able to take the different facets of annotations, which constitute a valuable context for document retrieval, into account.

As we have seen in Section 2.2, annotations and the referenced resources constitute a hypertext. This makes hypertext information retrieval approaches [4] potential candidates to be adapted to annotation-based retrieval. On the other hand, annotations might also be content about content such as reviews containing judgements about documents and thus cannot be seen as an extention of the document content. Nevertheless, such annotations contain information which are appropriate to take relevance criteria other than just topicality into account. Consider an example of a digital library where students could give judgements about documents, like "this book is a very good introduction", by annotating them. Another student might search for books which introduce her to the field of digital libraries. In this scenario we can see that the actual information 
need can be mapped onto two queries: One made to the set of documents for topicality (e.g., $q_{d o c}=$ "digital libraries"), and one made to the set of annotations (e.g., $q_{a n n}=$ "good introduction"). This results into a composed query $q=$ $\left(q_{d o c}, q_{a n n}\right)$. When seeing retrieval as uncertain inference, the retrieval weight of a document $d$ w.r.t the query $q$ is determined by the probability that $d$ implies $q$. A retrieval function calculating $P(d \rightarrow q)$ considering the annotation context of $d$ could roughly be outlined as, for example,

$$
P(d \rightarrow q)=\left(P_{d o c}\left(d \rightarrow q_{d o c}\right)+P_{a n n}\left(d \rightarrow q_{a n n}\right)-\Theta\right) \cdot\left(1-P_{a n n}\left(d \nrightarrow q_{a n n}\right)\right)
$$

$\Theta=P_{d o c}\left(d \rightarrow q_{d o c}\right) \cdot P_{a n n}\left(d \rightarrow q_{a n n}\right)$ reflects the possible jointness of events. The value for $P_{d o c}$ is determined by the weight of $d$ w.r.t. $q_{d o c}$ whereas the computation of $P_{a n n}$ is based on the annotations made on $d$. Negative annotations made on the document increase the probability $P_{a n n}\left(d \nrightarrow q_{a n n}\right)$ that the document does not imply the query and thus decrease $P(d \rightarrow q)$. The calculation of $P_{a n n}$ might need an in-depth analysis of the annotation thread as discussed in $[7]^{4}$. When seeing annotations as additional content rather than judgements, we do not need a composed query; in this case, it is $q=q_{d o c}=q_{a n n}$.

\section{Conclusions}

In this paper we have discussed the several ways in which digital libraries and collaboratories can benefit from annotations. We have shown different viewpoints on annotations, which can be seen as metadata, content, and dialogue acts. These realise a data, information and communication specific view on annotations. All our thoughts led to the presentation of annotation models covering a conceptual model and search and retrieval issues.

The proposed conceptual model for annotations is capable to represent the different viewpoints concerning annotations and enables the design and development of advanced retrieval functions. Furthermore it can be easily mapped to different models, such as a relational schema, an RDF schema or an XML schema, and it is suitable for developing an annotation service that can be seamlessly plugged into different digital libraries and collaboratories. Our considerations will be borne in mind for the specification and realisation of an annotation service within the BRICKS project ${ }^{5}$ which aims at establishing the organisational and technological foundations of a Digital Library at the level of a European Digital Memory.

With respect to annotations supporting access and retrieval in both digital libraries and collaboratories we have shown that there do not exist many retrieval models for annotation-based document retrieval. Our discussion of this resulted in an outline of a potential retrieval function based on the view of retrieval as uncertain inference. This function incorporates positive and negative evidence

\footnotetext{
${ }^{4}$ The proposed retrieval function can be seen as a generalisation of the one presented in [7].

${ }^{5}$ http://www. bricksfactory.org
} 
found both in the document content and in the according annotation thread. By applying such a retrieval function, relevance criteria other than topicality can be considered to satisfy users' information needs. Future research will discuss this issue more thoroughly and introduce suitable retrieval functions more precisely.

\section{Acknowledgements}

M. Agosti and N. Ferro were partially funded by ECD (Enhanced Contents Delivery), a joined program between the Italian National Research Council (CNR) and the Ministry of Education (MIUR), under the law 449/97-99. I. Frommholz and U. Thiel were partly funded by the 6th Framework IP BRICKS (IST-20022.3.1.12).

\section{References}

1. M. Agosti, L. Benfante, and N. Orio. IPSA: A Digital Archive of Herbals to Support Scientific Research. In T. M. T. Sembok, H. B. Zaman, H. Chen, S. R. Urs, and S. H. Myaeng, editors, Proc. 6th Int. Conf. on Asian Digital Libraries. Digital Libraries Digital Libraries: Technology and Management of Indigenous Knowledge (ICADL 2003), pages 253-264. LNCS 2911, Springer, Heidelberg, Germany, 2003.

2. M. Agosti and N. Ferro. Annotations: Enriching a Digital Library. In Koch and Sølvberg [12], pages 88-100.

3. M. Agosti and M. Melucci. Information Retrieval Techniques for the Automatic Construction of Hypertext. In A. Kent and C.M. Hall, editors, Encyclopedia of Library and Information Science, volume 66, pages 139-172. Marcel Dekker, New York, USA, 2000.

4. M. Agosti and A. Smeaton, editors. Information Retrieval and Hypertext. Kluwer Academic Publishers, Norwell (MA), USA, 1996.

5. M. Agosti. An Overview of Hypertext. In Agosti and Smeaton [4], pages 27-47.

6. H. Brocks, A. Stein, U. Thiel, I. Frommholz, and A. Dirsch-Weigand. How to incorporate collaborative discourse in cultural digital libraries. In Proc. of the ECAI 2002 Workshop on Semantic Authoring, Annotation \& Knowledge Markup (SAAKM02), Lyon, France, July 2002.

7. I. Frommholz, H. Brocks, U. Thiel, E. Neuhold, L. Iannone, G. Semeraro, M. Berardi, and $\mathrm{M}$. Ceci. Document-centered collaboration for scholars in the humanities - the COLLATE system. In Koch and Sølvberg [12], pages 434-445.

8. N. Fuhr, P. Hansen, M. Mabe, A. Micsik, and I. Sølvberg. Digital libraries: A generic classification and evaluation scheme. In Proc. of the 5th European Conf. on Research and Advanced Technology for Digital Libraries (ECDL2001), pages 187-199, Heidelberg et al., 2001. Springer.

9. M. Gertz, K.-U. Sattler, F. Gorin, M. Hogarth, and J. Stone. Annotating scientific images: A concept-based approach. In Proc. of the 14th Int. Conf. on Scientific and Statistical Database Management, pages 59-68. IEEE Computer Society, 2002.

10. G. Golovchinsky, M. N. Price, and B. N. Schilit. From reading to retrieval: Freeform ink annotations as queries. In F. Gey, M. Hearst, and R. Tong, editors, Proc. of the 22nd Annual Int. ACM SIGIR Conf. on Research and Development in Information Retrieval, pages 19-25, New York, 1999. ACM Press. 
11. J. Kahan and M.-R. Koivunen. Annotea: an open RDF infrastructure for shared Web annotations. In V. Y. Shen, N. Saito, M. R. Lyu, and M. E. Zurko, editors, Proc. 10th Int. Conf. on World Wide Web (WWW 2001), pages 623-632. ACM Press, New York, USA, 2001.

12. T. Koch and I. T. Sølvberg, editors. Proc. 7th European Conf. on Research and Advanced Technology for Digital Libraries (ECDL 2003). LNCS 2769, Springer, Heidelberg, Germany, 2003.

13. M.-R. Koivunen and E. Swick, R. Prud'hommeaux. Annotea Shared Bookmarks. http://www.w3.org/2001/Annotea/Papers/KCAP03/annoteabm.html, last visited 2004, March 18.

14. R.T. Kouzes, J.D. Myers, and W.A. Wulf. Collaboratories: Doing science on the Internet. IEEE Computer, 29(8), 1996.

15. C. C. Marshall and A. J. B. Brush. From Personal to Shared Annotations. In L. Terveen and D. Wixon, editors, Proc. Conf. on Human Factors and Computing Systems (CHI 2002) - Extended Abstracts, pages 812-813. ACM Press, New York, USA, 2002.

16. C. C. Marshall. Annotation: from Paper Books to the Digital Library. In R. B. Allen and E. Rasmussen, editors, Proc. 2nd ACM Int. Conf. on Digital Libraries (DL 1997), pages 233-240. ACM Press, New York, USA, 1997.

17. C. C. Marshall. Toward an Ecology of Hypertext Annotation. In R. Akscyn, editor, Proc. 9th ACM Conf. on Hypertext and Hypermedia (HT 1998): links, objects, time and space-structure in hypermedia systems, pages 40-49. ACM Press, New York, USA, 1998.

18. K. Nagao. Digital Content Annotation and Transcoding. Artech House, Norwood (MA), USA, 2003.

19. D. M. Nichols, D. Pemberton, S. Dalhoumi, O. Larouk, C. Belisle, and M. B. Twindale. DEBORA: Developing an interface to support collaboration in a digital library. In J.L. Borbinha and T. Baker, editors, Proc. of the 4 th European Conf. on Research and Advanced Technology for Digital Libraries (ECDL2000), pages 239-248. Springer: Berlin, 2000.

20. I. A. Ovsiannikov, M. A. Arbib, and T. H. McNeill. Annotation technology. Int. J. Hum.-Comput. Stud., 50(4):329-362, 1999.

21. T. Sannomiya, T. Amagasa, M. Yoshikawa, and S. Uemura. A framework for sharing personal annotations on web resources using XML. In M. E. Orlowska and M. Yoshikawa, editors, Proc. Workshop on Information Technology for Virtual Enterprises, pages 40-48. IEEE Computer Society Press, 2001.

22. J.R. Searle. A taxonomy of illocutionary acts. In J.R. Searle, editor, Expression and Meaning. Studies in the Theory of Speech Acts, pages 1-29. Cambridge University Press, Cambridge, 1979.

23. R. Wilensky. Digital library resources as a basis for collaborative work. J. Am. Soc. Inf. Sci., 51(3):228-245, 2000. 